\title{
Chemical Potential and the Nature of the Dark Energy: The case of phantom
}

\author{
J. A. S. Limat and S. H. Pereirat \\ Departamento de Astronomia, Universidade de São Paulo \\ Rua do Matão, 1226 - 05508-900, São Paulo, SP, Brazil
}

\begin{abstract}
The influence of a possible non zero chemical potential $\mu$ on the nature of dark energy is investigated by assuming that the dark energy is a relativistic perfect simple fluid obeying the equation of state (EoS), $p=\omega \rho(\omega<0$, constant). The entropy condition, $S \geq 0$, implies that the possible values of $\omega$ are heavily dependent on the magnitude, as well as on the sign of the chemical potential. For $\mu>0$, the $\omega$-parameter must be greater than -1 (vacuum is forbidden) while for $\mu<0$ not only the vacuum but even a phantomlike behavior $(\omega<-1)$ is allowed. In any case, the ratio between the chemical potential and temperature remains constant, that is, $\mu / T=\mu_{0} / T_{0}$. Assuming that the dark energy constituents have either a bosonic or fermionic nature, the general form of the spectrum is also proposed. For bosons $\mu$ is always negative and the extended Wien's law allows only a dark component with $\omega<-1 / 2$ which includes vacuum and the phantomlike cases. The same happens in the fermionic branch for $\mu<0$. However, fermionic particles with $\mu>0$ are permmited only if $-1<\omega<-1 / 2$. The thermodynamics and statistical arguments constrain the EoS parameter to be $\omega<-1 / 2$, a result surprisingly close to the maximal value required to accelerate a FRW type universe dominated by matter and dark energy $(\omega \lesssim-10 / 21)$.
\end{abstract}

PACS numbers: 98.80.-k, 95.36.+x

\section{INTRODUCTION}

The current idea of an accelerating Universe is based on a large convergence of independent observations, and its explanation constitutes one of the greatest challenges for our current understanding of fundamental physics [1, 2]. In the context of a Friedmann-Robertson-Walker (FRW) cosmology, dominated by pressureless matter with density $\rho_{m}$ plus an extra component of density $\rho$ and pressure $p$, the scale factor evolution is governed by the equation $3 \ddot{a} / a=-4 \pi G\left(\rho_{m}+\rho+3 p\right)$. This means that a hypothetical component with large negative pressure may drive the evolution of an expanding accelerating Universe. This exotic component is usually termed dark energy or quintessence, and it represents about $70 \%$ of the total content in the Universe. The origin and the nature of dark energy is still a mystery, however, there is no doubt that its existence is beyond the domain of the standard model of particle physics [3].

Among a number of possibilities to describe the dark energy component, the simplest and most theoretically appealing way is by means of a cosmological constant $\Lambda$, which acts on the FRW equations as an isotropic and homogeneous source with a constant equation of state parameter $p / \rho=-1$. On the other hand, although cosmological scenarios with a $\Lambda$ term might explain most of the current astronomical observations, from the theoretical viewpoint they are plagued with some fundamental problems thereby stimulating the search for alternative dark energy models driven by different candidates [4, 5].

In the XCDM scenario, the dark energy component

\footnotetext{
*Electronic address: limajas@astro.iag.usp.br

${ }^{\dagger}$ Electronic address: spereira@astro.iag.usp.br
}

is phenomenologically described by an equation of state $p=\omega \rho$. The case $\omega=-1$ reduces to the cosmological constant. The imposition $\omega \geq-1$ is physically motivated by the classical fluid description [6]. This hyphothesis introduces a strong bias in the $\omega$-parameter determination from observational data. In order to circunvent such a difficulty, superquintessence or phantom dark energy cosmologies have been recently considered where such a condition is relaxed [7]. In contrast to the usual quintessence model, a decoupled phantom component presents an anomalous evolutionary behavior. For instance, the existence of future curvature singularities, a growth of the energy density with the expansion, or even the possibility of a rip-off of the structure of matter at all scales are theoretically expected. Although possessing such strange features, the phantom behavior is theoretically allowed by some kinetically scalar field driven cosmology [8], as well as, by brane world models [9], and, perhaps, more important to the present work, a PhantomCDM cosmology is not ruled out by the present type Ia Supernovae and other observations [10, 11].

In a series of papers [12, 13], we have studied some thermodynamics and statistical properties of dark energy with no chemical potential $(\mu=0)$. By using standard thermodynamics for a relativistic simple fluid, we concluded that the case of phantom energy is ruled out because the total entropy of a dark component with $\omega<-1$ is negative. In addition, by combining thermodynamics and statistical arguments the EoS was restricted to the interval $-1 \leq \omega<-1 / 2$ and a fermionic nature to the dark energy particles was favored.

Later on, thermodynamics arguments in favor of the phantom hypothesis were put forward by González-Díaz and Sigüenza 14]. They claimed that the temperature of a phantomlike fluid is always negative in order to keep its entropy positive definite (as statistically required). This 
new viewpoint was justified by arguing that the scalar field representation of a phantom field has a negative kinetic term $\dot{\phi}^{2}$ which quantifies the translational kinetic energy of the associated fluid system, and, as such, its temperature (a measure of the average kinetic energy) should be negative. Although temptative to some degree, both approaches have been considered in the literature (see, for instance, [15, 16] and Refs. therein). More important to the present work, they share a common property, namely, the chemical potential of the dark energy fluid was set to be zero from the very beginning.

In this article we reanalyze the thermodynamic and statistical properties of the dark energy scenario by considering the existence of a non-zero chemical potential. In order to clarify some subtleties present in the earlier results, we rederive the physical quantities in the presence of $\mu$ by adopting the full thermostatistic approach proposed in Refs. [12, 13]. This means that all thermodynamic and statistical properties follow directly from the EoS plus the hypothesis that $\mu \neq 0$. In particular, the temperatures of the dark energy fluids must be always positive definite. This is an interesting aspect of the present work since there are many scalar field representations for dark energy fluids, however, the thermodynamic laws are independent to what happens at a microscopic level as long as the equation of state has been defined. As we shall see, one of the main consequences of a negative chemical potential is that the phantom scenario is recovered without the need to appeal to negative temperatures. In addition, a bosonic or fermionic nature of the dark energy component now becomes possible.

The paper is planned as follows. In section 2 we discuss the thermodynamic constraints when a chemical potential is introduced. In section 3 we consider a statistical analysis by assuming that the dark energy particles are massless and can have either a bosonic or a fermionic nature. In the conclusion section, a joint analysis involving the thermodynamic and statistical constraints on the EoS $\omega$-parameter is presented

\section{COSMOLOGY, DARK ENERGY AND THERMODYNAMICS}

Let us now consider that the Universe is described by the homogeneous and isotropic Friedmann-RobertsonWalker (FRW) geometry $(c=1)$

$$
d s^{2}=d t^{2}-a^{2}(t)\left(\frac{d r^{2}}{1-\kappa r^{2}}+r^{2} d \theta^{2}+r^{2} \sin ^{2} \theta d \phi^{2}\right),
$$

where $\kappa=0, \pm 1$ is the curvature parameter and $a(t)$ is the scale factor. In what follows we consider that the matter content (or at least one of its noninteracting components is a fluid described by the EoS

$$
p=\omega \rho,
$$

where $p$ is the pressure, $\rho$ is the energy density and $\omega$ a constant parameter which may be positive (white energy) and negative (dark energy). The cases $\omega=1 / 3,1$, and -1 characterizes, respectively, the blackbody radiation, a stiff-fluid and the vacuum state while $\omega<-1$ stands to a phantomlike behavior.

Following standard lines, the equilibrium thermodynamic states of a relativistic simple fluid are characterized by an energy momentum tensor $T^{\alpha \beta}$, a particle current $N^{\alpha}$ and an entropy current $S^{\alpha}$ which satisfy the following relations

$$
\begin{gathered}
T^{\alpha \beta}=(\rho+p) u^{\alpha} u^{\beta}-p g^{\alpha \beta}, \quad T_{; \beta}^{\alpha \beta}=0, \\
N^{\alpha}=n u^{\alpha}, \quad N^{\alpha} ; \alpha=0 \\
S^{\alpha}=s u^{\alpha}, \quad S^{\alpha} ;_{\alpha}=0
\end{gathered}
$$

where (;) means covariant derivative, $n$ is the particle number density, and $s$ is the entropy density. In the FRW background, the above conservation laws can be rewritten as (a dot means comoving time derivative)

$$
\dot{\rho}+3(1+\omega) \rho \frac{\dot{a}}{a}=0, \dot{n}+3 n \frac{\dot{a}}{a}=0, \dot{s}+3 s \frac{\dot{a}}{a}=0,
$$

whose solutions can be written as:

$$
\rho=\rho_{0}\left(\frac{a_{0}}{a}\right)^{3(1+\omega)}, n=n_{0}\left(\frac{a_{0}}{a}\right)^{3}, s=s_{0}\left(\frac{a_{0}}{a}\right)^{3},
$$

where the positive constants $\rho_{0}, n_{0}, s_{0}$ and $a_{0}$ are the present day values of the corresponding quantities (hereafter present day quantities will be labeled by the index "0"). On the other hand, the quantities $p, \rho, n$ and $s$ are related to the temperature $T$ by the Gibbs law

$$
n T d\left(\frac{s}{n}\right)=d \rho-\frac{\rho+p}{n} d n,
$$

and from Gibbs-Duhem relation [18 there are only two independent thermodynamic variables, say, $n$ and $T$. Now, by assuming that $\rho=\rho(T, n)$ and $p=p(T, n)$ and combining the thermodynamic identity [17]

$$
T\left(\frac{\partial p}{\partial T}\right)_{n}=\rho+p-n\left(\frac{\partial \rho}{\partial n}\right)_{T}
$$

with the conservation laws as given by (6), one may show that the temperature satisfies

$$
\frac{\dot{T}}{T}=\left(\frac{\partial p}{\partial \rho}\right)_{n} \frac{\dot{n}}{n}=-3 \omega \frac{\dot{a}}{a} .
$$

Therefore, assuming that $\omega \neq 0$ we obtain

$$
n=n_{0}\left(\frac{T}{T_{0}}\right)^{\frac{1}{\omega}} \Leftrightarrow T=T_{0}\left(\frac{a}{a_{0}}\right)^{-3 \omega}
$$


The temperatures appearing in the above expressions are positive regardless of the value of $\omega$. In particular, in the radiation case $(\omega=1 / 3)$, one finds $a T=a_{0} T_{0}$ as should be expected. As compared to this case, the unique difference is that the dark energy fluid (even in the phantom regime) becomes hotter in the course of the cosmological adiabatic expansion since its equation-of-state parameter is a negative quantity. A physical explanation for this behavior is that thermodynamic work is being done on the system [12, 13].

It should be stressed that the derivation of the temperature evolution law presented here is fully independent of the entropy function, as well as, of the chemical potential $\mu$. The above expressions also imply that for any comoving volume of the fluid, the product $T^{\frac{1}{\omega}} V$ remains constant in the course of expansion and must also characterize the equilibrium states (adiabatic expansion) regardless of the value of $\mu$. Further, by inserting the temperature law into the energy conservation law (7), one obtains the energy density as function of the temperature

$$
\rho=\rho_{0}\left(\frac{T}{T_{0}}\right)^{\frac{1+\omega}{\omega}} .
$$

Now, in order to determine the chemical potential and its influence on the thermodynamics of dark energy, we consider the Euler relation [18]

$$
T s=p+\rho-\mu n,
$$

where $\mu$ in general can also be a function of $T$ and $n$ 19, 20]. By combining the above expression with equations (21), (71) and (11) we obtain:

$$
\mu=\mu_{0}\left(\frac{a}{a_{0}}\right)^{-3 \omega}=\mu_{0}\left(\frac{T}{T_{0}}\right)
$$

where

$$
\mu_{0}=\frac{1}{n_{0}}\left[(1+\omega) \rho_{0}-T_{0} s_{0}\right]
$$

This straightforward thermodynamic result has some interesting consequences. In principle, the chemical potential may be either positive or negative, and it also depends on the values of the EoS $\omega$-parameter. In particular, $\mu$ is always negative $\left(\mu_{0}<0\right)$ in the case of phantom energy, and becomes even more negative in the course of time ( $T$ grows with the scale factor during the cosmic evolution). It is also known that $\mu$ is zero in the case of photons $(\omega=1 / 3)$ because they are their own antiparticles [21]. In this case, (15) yields correctly that $3 s_{0} T_{0}=4 \rho_{0}$ as should be expected. In general, if $\mu=0$, necessarily the relation $s_{0} T_{0}=(1+\omega) \rho_{0}$ must be obeyed, which is just the present day expression of $s T=(1+\omega) \rho$ as required by (13).
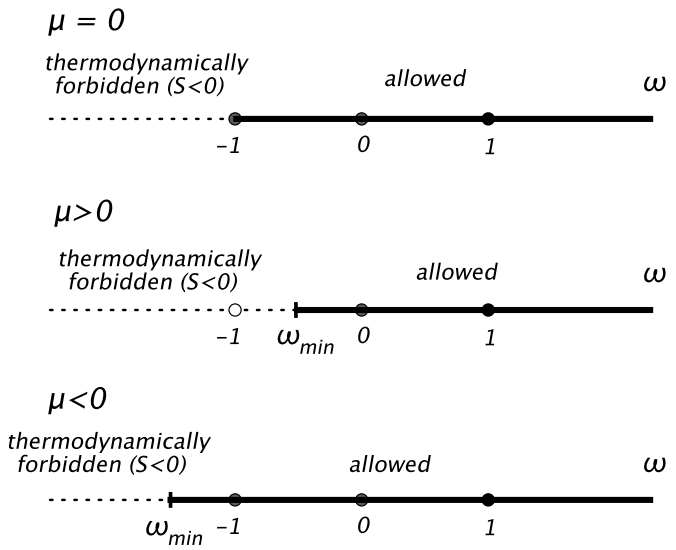

FIG. 1: The allowed intervals of $\omega$ values (heavy lines) and forbidden (dashed lines) for null, positive and negative chemical potentials. Note that a large portion of the dark branch $\omega<0$ is always thermodynamically permitted. However, for $\mu \geq 0$, the phantomlike behavior $(\omega<-1)$ is thermodynamically forbidden.

At this point, the fundamental question is: How the chemical potential modifies the entropy constraints 12 , 13] derived in the previous papers?

In order to show that we compute explicitly the entropy of dark energy for a comoving volume $V$. As remarked before, the entropy function should scale as $S \propto T^{\frac{1}{\omega}} V$. Actually,

$S(T, V) \equiv s V=\left[\frac{(1+\omega) \rho_{0}-\mu_{0} n_{0}}{T_{0}}\right]\left(\frac{T}{T_{0}}\right)^{1 / \omega} V=s_{0} V_{0}$,

which remains constant as expected (see discussion below Eq.(11)). However, in order to keep the entropy $S \geq 0$ (as statistically required), the following constraint must be satisfied:

$$
\omega \geq \omega_{\min }=-1+\frac{\mu_{0} n_{0}}{\rho_{0}},
$$

which introduces a minimal value to the $\omega$-parameter, below which the entropy becomes negative. This is a remarkable expression and its consequences are apparent. For instance, consider that $\mu_{0}=0$ (no chemical potential). In this case, the smallest value of the $\omega$-parameter is $\omega_{\min }=-1$ and the previous analysis by Lima and Alcaniz [13] is fully recovered, that is, the phantom domain $(\omega<-1)$ is thermodynamically forbidden. However, for a negative chemical potential, the phantomlike regime becomes thermodynamically allowed thereby recovering the hypothesis of phantom energy without appealing to negative temperature as proposed in the literature [14]. Note also that for a positive chemical potential not even a cosmological constant $(\omega=-1)$ is possible. In figure 1 , we summarize the basic thermodynamic results. 

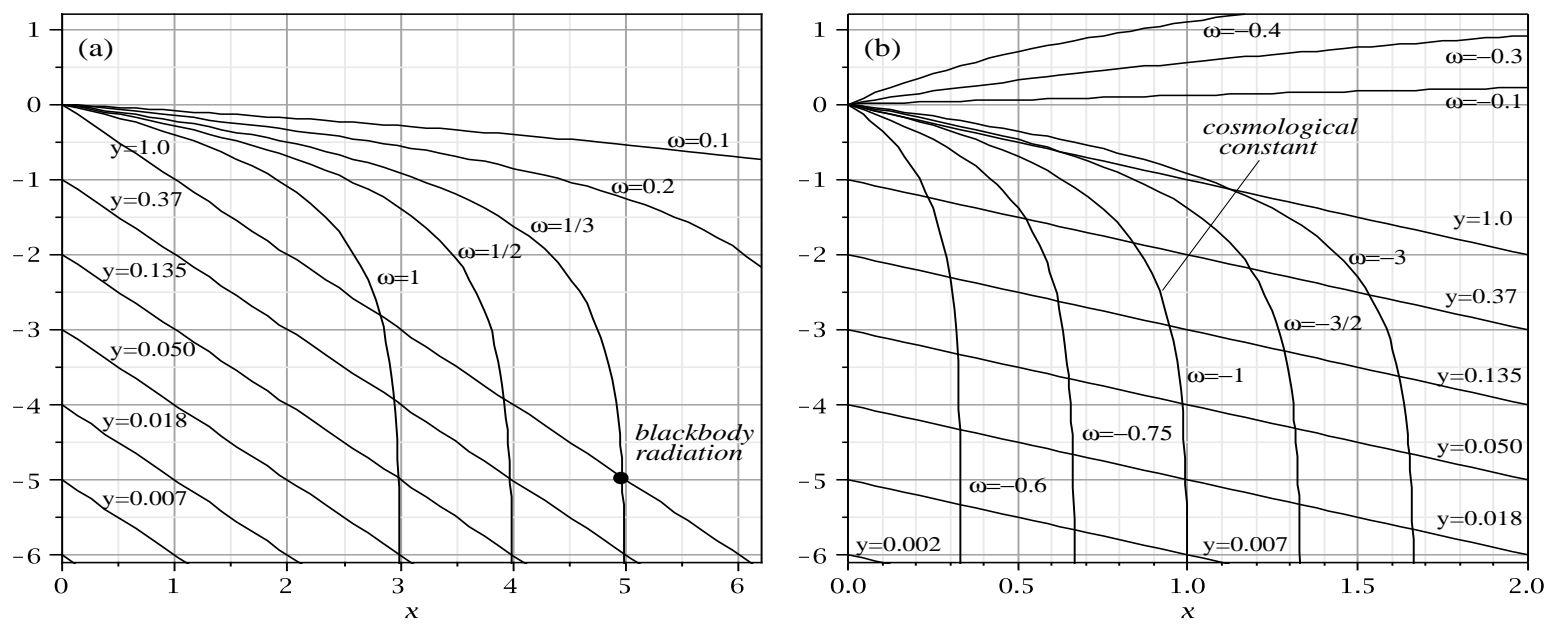

FIG. 2: Solutions for the bosonic case $(\mu \leq 0)$. a) For given values of the pair $(\mathrm{x}, \mathrm{y})$, the straight lines and curves represent the l.h.s. side and the r.h.s. of (21), respectively. The intersection points between the curves and the straight lines represent the desired physical solutions for $\omega>0$. The standard radiation solution $(\mu=0$ and $\omega=1 / 3)$ is indicated by a black point. b) Curves representing the right hand side of (21) for different $\omega$ values in the region $\omega<-1 / 2$ (lower half plane) and $-1 / 2<\omega \leq 0$ (upper half plane). The cosmological constant case $(\omega=-1)$ is indicated. For both diagrams the fugacity $y=\exp \left(\mu / k_{B} T\right) \leq 1$.

\section{DARK ENERGY WITH A CHEMICAL POTENTIAL: STATISTICAL BEHAVIOR}

Another interesting feature of a dark energy component with a non zero chemical potential is related to its spectral distribution. The generalized Wien-type spectrum for dark energy with $\mu=0$ has already been discussed in the literature [12, 13]. A different approach for the phantomlike behavior involving the modulus of the temperature has also been proposed [14]. In the present case, since the temperature is positive, we simply add the chemical potential $\mu$ to the spectrum previously derived [12, 13]. More precisely, we postulate the following spectral distribution:

$$
\rho(T, \nu)=\frac{\alpha \nu^{1 / \omega}}{\mathrm{e}^{(h \nu-\mu) / k_{B} T}+\epsilon},
$$

where $\epsilon=+1$ stands for the Fermi-Dirac distribution and $\epsilon=-1$ to the Bose-Einstein one, and $\alpha$ is a positive and $\omega$-dependent constant. Here it is important to note that for bosons the chemical potential is always negative or null, while for fermions it may be positive or negative [21].

A direct consequence of (18) is related to the displacement Wien's law. The wavelength for which the distribution attains its maximum value is determined by the condition

$$
\lambda_{m} T=\frac{h c}{k_{B} x^{\prime}(\omega)}=\frac{1.438}{x^{\prime}(\omega)},
$$

where $x^{\prime}(\omega)$ is the root of the transcendent equation

$$
y \mathrm{e}^{-x}=-\epsilon+\left[\frac{\epsilon \omega}{1+2 \omega}\right] x,
$$

where $x=h c / k_{B} \lambda T$ and $y=\exp \left(\mu / k_{B} T\right) \equiv$ $\exp \left(\mu_{0} / k_{B} T_{0}\right)$ is a constant fugacity. When $\mu_{0}=0$ the above expressions reduces to the one obtained in [13]. The solution of the above algebraic equation can be derived both numerically and graphically. We are only interested in solutions with positive $x$, because the temperature is always positive. Due to the presence of the chemical potential, the analysis of the above condition will be done separately for bosons and fermions.

\section{A. Bosons}

Let us now analyze the bosonic case $(\epsilon=-1)$. It proves convenient to rewrite condition (20) in the following form:

$$
\ln y-x=\ln \left(1-\frac{\omega x}{1+2 \omega}\right)
$$

For each value of $\mu$, the left hand side (1.h.s.) of the above expression is a collection of straight lines with slope equal to -1 . Since $\mu$ is always negative or null for bosons, it follows that $0<y \leq 1$ so that $-\infty<\ln y \leq 0$. This means that the 1.h.s. of (21) is a collection of parallel straight lines on the lower half plane. The first straight line is the trivial solution with zero chemical potential.

Note also that the right hand side (r.h.s.) of (21) involves the singularity for $\omega=-1 / 2$, and, as such, must 


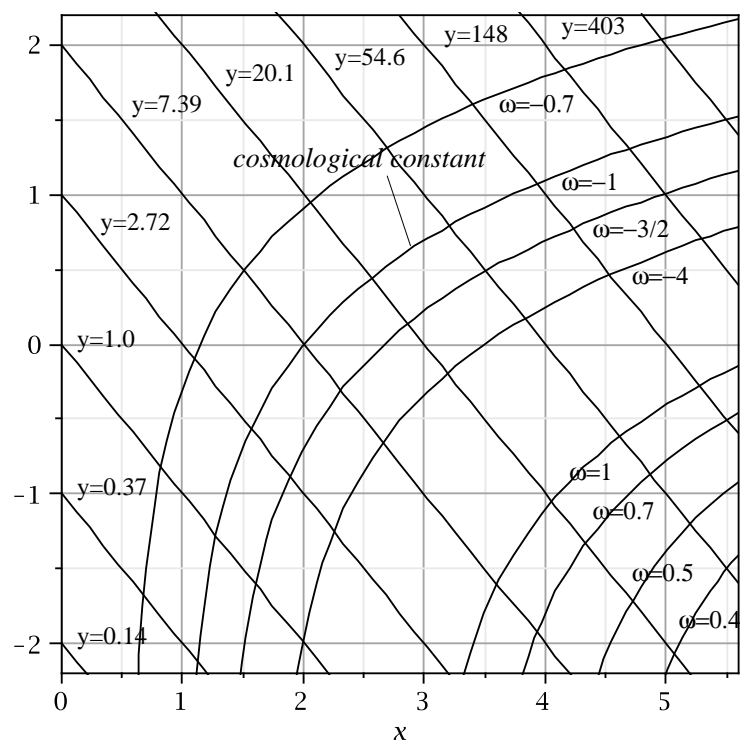

FIG. 3: Curves representing the right hand side of (22), for different $\omega$ values in the region $\omega<-1 / 2$ and $\omega>0$.

be separately analyzed. Apart from this point, we have 3 different intervals, namely: $\omega>0, \omega<-1 / 2$ and $-1 / 2<\omega \leq 0$.

In Figures $2 \mathrm{a}$ and $2 \mathrm{~b}$ we display the main results. When $\omega>0$ we have a collection of curves represented in Fig. 2a. All of them cross the straight lines in some point $x>0$ thereby indicating the solutions of the algebraic equation (21). The standard radiation solution ( $\mu=0$ and $\omega=1 / 3$ ) is indicated, however, it should also be remarked the theoretical possibility of a radiation solution with $\mu \neq 0$. For $\omega<-1 / 2$ we have the collection of curves represented on the lower half plane of Figure 2b, superposed to the straight lines. This analysis show that all these curves cross the straight lines in some positive $x$ value, indicating a solution to the algebraic expression (21). Finally, on the interval $-1 / 2<\omega \leq 0$ we have the set of curves represented on the upper half plane of Figure $2 \mathrm{~b}$ thereby showing the absence of physical solutions.

In summ, a simple graphic analysis shows that there are two intervals of $\omega$ for which the condition (21) has a solution, namely, $\omega>0$ and $\omega<-1 / 2$, while the interval $-1 / 2<\omega \leq 0$ is statistically forbidden. Therefore, unlike the previous analyzes with $\mu=0$ [12, 13], the EoS $\omega<-1 / 2$ for bosons now becomes possible when the chemical potential is negative. This includes the phantom dark energy as a physical possibility.

\section{B. Fermions}

The analysis of the fermionic case $(\epsilon=+1)$ is similar to the bosonic one, but now the chemical potential can be either positive or negative. The condition (21) for the
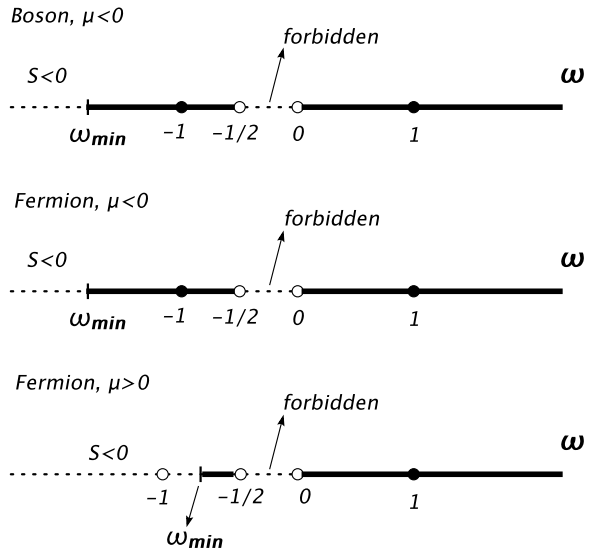

FIG. 4: Thermodynamic and statistical constraints. The allowed (heavy lines) and forbidden (dashed lines) values of $\omega$ for the bosonic and fermionic cases with $\mu<0$ and $\mu>0$. The phantom branch $\omega<-1$ is excluded for the fermionic case with $\omega>0$. Note that the dark branch $-1<\omega<-1 / 2$ for bosons is now possible.

fermionic case reads

$$
\ln y-x=\ln \left(-1+\frac{\omega x}{1+2 \omega}\right) .
$$

The analysis on the 1.h.s. of (22) is similar to the bosonic case, the unique difference is that $\mu_{0}$ can be positive. In this case, we have $y>1$ and $\ln y>0$. As in the previous case, the discussion on the r.h.s. of (22) depends on the $\omega$ values. For the cases $\omega<-1 / 2$ and $\omega>0$ we have the curves represented in Figure 3. We see that all curves crossing the straight lines for some positive value of $x$ yield a valid solution for (22). Note also that on the interval $-1 / 2<\omega<0$, all the curves are in the negative $x$-axis (negative temperatures), and, therefore, none of them cross the straight lines (two reasons for the the interval be a forbidden region).

\section{CONCLUDING REMARKS}

In this paper we have investigated the thermodynamic and statistical properties of a dark energy fluid with equation of state, $p=\omega \rho$, by assuming that its chemical potential is different from zero.

In Figure 4, we summarize the main results of the present analysis by combining both approaches. As discussed in section 2, the regions with $S<0$ are thermodynamically forbidden. Note also that many dark energy fluids satisfy the combined constraints regardless of the $\mu$ sign, that is, a large interval of negative $\omega$ values is allowed by thermodynamic and statistical considerations. However, a phantom like behavior $(\omega<-1)$ is permitted only for $\mu<0$, and the corresponding massless particles can have either a bosonic or fermionic nature. 
It was also proved (see also Fig. 4) that the EoS parameter of a dark energy fluid obeying a generalized Wien's law always satisfies the constraint $\omega<-0.5$ (a thermostatistics limit). This upper limit is surprisingly close to the maximal value of the EoS $\omega$-parameter necessary to accelerate the present universe. Actually, in order to accelerate a FRW universe dominated by matter and dark energy, the EoS parameter must satisfies the inequality, $\omega<-\left(1+\Omega_{m} / \Omega_{x}\right) / 3$. Therefore, for $\Omega_{m} \cong 0.3$ and $\Omega_{x} \cong 0.7$, as indicated by the present observations [1, 2], one finds the dynamic constraint $\omega \lesssim-10 / 21$.

Finally, it should be stressed that for $\mu=0$ one finds $\omega_{\text {min }}=-1$ (see Eq. (17)) in accordance to the results previously derived by Lima and Alcaniz [13]. The present analysis with $\mu \neq 0$ also opens the possibility for an EoS parameter $\omega<-1$, thereby recovering the idea of a phantom dark energy without to appeal to negative temper- atures. Perhaps more interesting, unlike the results for $\mu=0$ which favored only a fermionic nature to the dark energy fluid (phantom and nonphantom), it was demonstrated that a bosonic kind of dark energy becomes possible from a thermostatistics viewpoint.

\section{Acknowledgments}

The authors would like to thank V. C. Busti, J. V. Cunha, J. F. Jesus, A. C. Guimaraes, R. Holanda and R. C. Santos for helpful discussions. JASL is partially supported by CNPq and FAPESP No. 04/13668-0 and SHP is supported by CNPq No. 150920/2007-5 (Brazilian Research Agencies).
[1] A. G. Riess et al., Astron. J. 116, 1009 (1998); S. Perlmutter et al., Astrophys. J. 517, 565 (1999); P. Astier et al., Astron. Astrophys. 447, 31 (2006); A. G. Riess et al., Astro. J. 659, 98 (2007).

[2] D. N. Spergel et al. Astrophys. J. Suppl. Ser. 170, 377 (2007); S. W. Allen et. al., arXiv:0706.0033v1 (2007).

[3] T. Padmanabhan, Phys. Rept. 380, 235 (2003); P. J. E. Peebles and B. Ratra, Rev. Mod. Phys. 75, 559 (2003); J. A. S. Lima, Braz. Jour. Phys. 34, 194 (2004), astro-ph/0402109; V. Sahni and A. Starobinsky, IJMP D15, 2105 (2006).

[4] S. Weinberg, Rev. Mod. Phys. 61, 1 (1989); D. Pavón, Phys. Rev. D 43, 375 (1991); J. C. Carvalho, J. A. S. Lima and I. Waga, Phys. Rev. D 46, 2404 (1992); J. A. S. Lima and J. M. F. Maia, Phys. Rev D 49, 5597 (1994); J. A. S. Lima and M. Trodden, Phys. Rev. D 53, 4280 (1996), astro-ph/9508049]; J. A. S. Lima, Phys. Rev. D 54, 2571 (1996), gr-qc/9605055; A. I. Arbab and A. M. M. Abdel-Rahman, Phys. Rev. D 50, 7725 (1994); J. M. Overduin and F. I. Cooperstock, Phys. Rev. D 58, 043506 (1998); R. G. Vishwakarma, Class. Quant. Grav. 17, 3833 (2000); M. V. John and K. B. Joseph, Phys. Rev. D 61, 087304 (2000); J. A. S. Lima and J. S. Alcaniz Mon. Not. Roy. Astron. Soc. 317, 893 (2000), astro-ph/0005441; J. M. F. Maia and J. A. S. Lima, Phys. Rev. D 65, 083513 (2002), astro-ph/0112091; M. K. Mak, J.A. Belinchon, and T. Harko, IJMPD 11, 1265 (2002); M. R. Mbonye, IJMPA 18, 811 (2003); J. V. Cunha and R. C. Santos, IJMP D 13, 1321 (2004), astro-ph/0402169]; S. Carneiro and J. A. S. Lima, IJMPA 202465 (2005), gr-qc/0405141; I. L. Shapiro, J. Sola and H. Stefancic, JCAP 0501, 012 (2005); E. Elizalde, S. Nojiri, S.D. Odintsov and P. Wang, Phys. Rev. D 71, 103504 (2005); J. S. Alcaniz and J. A. S. Lima, Phys. Rev. D 72, 063516 (2005), astro-ph/0507372; A. Friaca, J. S. Alcaniz and J. A. S. Lima, Mon. Not. R. Astron. Soc. 362, 1295 (2005), astro-ph/0504031.

[5] T. Padmanabhan and T. R. Choudhury, Mon. Not. Roy. Astron. Soc. 344, 823 (2003); P. T. Silva and O. Bertolami, Astrophys. J. 599, 829 (2003); J. A. S. Lima, J.
V. Cunha and J. S. Alcaniz, Phys. Rev. D 68, 023510 (2003), astro-ph/0303388; Z.-H. Zhu and M.-K. Fujimoto, Astrophys. J. 585, 52 (2003); L. P. Chimento, Phys. Rev.D 69, 123517 (2004); S. Nesseris and L. Perivolaropoulos, Phys. Rev. D 70, 043531 (2004); Y. Wang and M. Tegmark, Phys. Rev. Lett. 92, 241302 (2004); T. R. Choudhury and T. Padmanabhan, Astron. Astrophys. 429807 (2005); D. Rapetti, S. W. Allen and J. Weller, Mon. Not. Roy. Astron. Soc. 360, 546 (2005); R. Colistete Jr and J. C. Fabris, Class. Quant. Grav. 22, 2813 (2005); B. Feng, X. Wang and X. Zhang, Phys. Lett. B 607, 35 (2005); R. Lazkoz, S. Nesseris and L. Perivolaropoulos, astro-ph/0503230; A. Shafieloo, U. Alam, V. Sahni, A. A. Starobinsky, Mon. Not. Roy. Astron. Soc. 366, 1081 (2006); J. V. Cunha, J. S. Alcaniz and J. A. S. Lima, Phys. Rev. D 69, 083501 (2004), astro-ph/0306319]; F. C. Carvalho et al., Phys. Rev. Lett. 97, 081301 (2006), astro-ph/0608439; arXiv:0704.3043; L. R. Abramo and N. Pinto-Neto, Phys. Rev. D 73, 063522 (2006); J. V. Cunha, L. Marassi and R. C. Santos, IJMP D 16, 403 (2007), astro-ph/0306319.

[6] S. W. Hawking and G. F. R. Ellis, The large scale structure of space-time, Cambridge UP, Cambridge (1973).

[7] V. Faraoni, IJMP D 11, 471 (2002); R. R. Caldwell, M. Kamionkowski, N. N. Weinberg, PRL 91, 071301 (2003); J. Santos and J. S. Alcaniz, Phys. Lett. B 619, 11 (2005); E. V. Linder, arXiv:0704.2064. (2007).

[8] T. Chiba, T. Okabe, M. Yamaguchi, Phys. Rev. D 62, 023511 (2000).

[9] V. Shani and Y. Shtanov, IJMPA 11, 1 (2002); V. Shani and Y. Shtanov, JCAP 0311, 014 (2003), astro-ph/0202346; S.-F. Wu, A. Chatrabhuti, G.-H. Yang and P-M Zhang, arXiv:0708.1038. (2007).

[10] T. R. Choudhury and T. Padmanabhan, Phys. Rev. D 69, 064033 (2004).

[11] Y-S Piao and Y-Z Zhang, Phys. Rev. D 70, 063513 (2004); T. R. Choudhury and T. Padmanabhan, ASP Conference Series 342, 497 (2005); Astron. and Astrophys. 429, 807 (2005); R. C. Santos and J. A. S. Lima (2006) astro-ph/0609129; L. Perivolaropoulos, 
Phys. Rev. D 71, 063503 (2005); S. Nesseris and L. Perivolaropoulos, JCAP 0701, 018 (2007).

[12] J. A. S. Lima and A. Maia Jr., Phys. Rev. D 52, 5628 (1995); ibdem, Int. J. Theor. Phys, 34, 9 (1995), gr-qc/9505052; J. A. S. Lima and J. Santos, Int. J. Theor. Phys. 34, 143 (1995); J. A. E. Carrillo, J. A. S. Lima, A. Maia Jr., Int. J. Theor. Phys. 35, 2013 (1996). hep-th/9906016.

[13] J. A. S. Lima and J. S. Alcaniz, Phys. Lett. B 600, 191 (2004). astro-ph/0402265

[14] P. F. González-Díaz and C. L. Sigüenza, Nucl. Phys. B 697,363 (2004); Phys. Lett.B 589, 78 (2004).

[15] G. Izquerdo and D. Pavón, Phys. Lett. B 600, 420 (2006).

[16] J. A. Freitas Pacheco and J. E. Horvath, Class. Quant. Grav. 24, 5427 (2007).
[17] S. Weinberg, Astrop. J. 168, 175 (1971); R. Silva, J. A. S. Lima and M. O. Calvão, Gen. Rel. Grav. 34, 865 (2002), gr-qc/0201048

[18] H. B. Callen, Thermodynamics and an Introduction to Thermostatistics, 2nd Edition, Wiley, New York (1985).

[19] In the case of a relativistic ideal gas of massive particles, for instance, the so-called fugacity reads, $\exp \left(\mu / k_{B} T\right)=$ $n(2 \pi \bar{h})^{3} / 4 \pi m^{2} c k_{B} K_{2}\left(m c^{2} / k_{B} T\right)$, where $m$ is the particles mass and $K_{2}$ is the modified Bessel of second kind.

[20] S. R. de Groot, W. A. van Leewen and Ch. G. van Weert, Relativistic Kinetic Theory, North Holland, Amsterdam (1980).

[21] L. D. Landau and E. M. Lifschitz, Statistical Physics, Part 1, Pergamon Press, Oxford (1980). 